\title{
Hippocampal synaptic and membrane function in the DBA/2J-mdx mouse model of Duchenne muscular dystrophy
}

Article

Accepted Version

Creative Commons: Attribution-Noncommercial-No Derivative Works 4.0

Bianchi, R., Eilers, W., Pellati, F., Corsi, L., Foster, H., Foster, K. and Tamagnini, F. (2020) Hippocampal synaptic and membrane function in the DBA/2J-mdx mouse model of Duchenne muscular dystrophy. Molecular and Cellular Neuroscience, 104. 103482. ISSN 1044-7431 doi: https://doi.org/10.1016/j.mcn.2020.103482 Available at https://centaur.reading.ac.uk/89429/

It is advisable to refer to the publisher's version if you intend to cite from the work. See Guidance on citing.

To link to this article DOI: http://dx.doi.org/10.1016/j.mcn.2020.103482

Publisher: Elsevier

All outputs in CentAUR are protected by Intellectual Property Rights law, including copyright law. Copyright and IPR is retained by the creators or other copyright holders. Terms and conditions for use of this material are defined in the End User Agreement. 


\section{CentAUR}

Central Archive at the University of Reading

Reading's research outputs online 
1 Hippocampal synaptic and membrane function in

\section{muscular dystrophy}

Abbreviated title: Duchenne's associated hippocampal hypoexcitability

Key words: Membrane properties, Duchenne's muscle dystrophy, Hippocampus, Synaptic function, after-hyperpolarization.

Authors: Riccardo Bianchi ${ }^{1,2}$, Wouter Eilers ${ }^{3}$, Federica Pellati ${ }^{2}$, Lorenzo Corsi², Helen Foster ${ }^{3}$, Keith Foster ${ }^{3}$ \& Francesco Tamagnini ${ }^{1}$

${ }^{1}$ University of Reading School of Pharmacy, Hopkins Building, Whiteknight Campus, Reading, RG6 6LA, UK.

2 University of Modena and Reggio Emilia, Department of Life Sciences, Via G. Campi 103287, 41125 Modena, Italy

${ }^{3}$ University of Reading School of Biological Sciences, Hopkins Building, Whiteknight Campus, Reading, RG6 6LA, UK.

Corresponding author: Dr Francesco Tamagnini Email: F.Tamagnini@reading.ac.uk T: +44 (0)118378 4745 
Highlights

- Duchenne's muscular dystrophy is often associated with mental retardation

- The association between dystrophin and excitatory neuronal function in hippocampal physiology is yet largely unexplored

- We show that hippocampal function is largely preserved in the dystrophinlacking DBA/2J-mdx mouse model

- Medium after-hyperpolarization is increased in the DBA/2J-mdx mouse model

Abstract

Dystrophin deficiency is associated with alterations in cell physiology. The functional consequences of dystrophin deficiency are particularly severe for muscle physiology, as observed in Duchenne muscle dystrophy (DMD). DMD is caused by the absence of a $427 \mathrm{kDa}$ isoform of dystrophin. However, in addition to muscular dystrophy symptoms, DMD is frequently associated with memory and attention deficits and epilepsy. While this may be associated with a role for dystrophin in neuronal physiology, it is not clear what neuronal alterations are linked with DMD. Our work shows that CA1 pyramidal neurons from DBA/2J$m d x$ mice have increased afterhyperpolarization compared to WT controls. All the other electrotonic and electrogenic membrane properties were unaffected by this genotype. Finally, basal synaptic transmission, short-term and long-term synaptic plasticity at Schaffer collateral to CA1 glutamatergic synapses were unchanged between $m d x$ and WT controls. These data show that the excitatory component of hippocampal activity is largely preserved in DBA/2J$m d x$ mice. Further studies, extending the investigation to the inhibitory GABAergic function, may provide a more complete picture of the functional, network alterations underlying impaired cognition in DMD. In addition, the investigation of changes in neuronal single conductance biophysical properties associated with this genotype, is required to identify the functional alterations associated with dystrophin deficiency and clarify its role in neuronal function. 
57 The $427 \mathrm{kDa}$ isoform of dystrophin (Dp427) ${ }^{1}$, is present in healthy striated/smooth muscle and neurons, particularly in the hippocampus, prefrontal cortex and cerebellum ${ }^{2,3}$. The lack of dystrophin causes Duchenne muscular dystrophy (DMD) which involves progressive wasting of skeletal muscle and the replacement of muscle tissue with connective and adipose tissue, as well as the development of dilated cardiomyopathy in later stages of the disease ${ }^{4,5}$. In addition, DMD has been associated with altered brain function, including attention deficits, memory impairments and increased risk of seizures, which suggests a role for dystrophin in brain function ${ }^{6}$. In the brain, neurons expressing the Dp427 isoform include cerebellar Purkinje cells, hippocampal and cortical pyramidal cells and cells in the amygdala ${ }^{7-10}$; other brain isoforms are Dp140 and the Dp71 ${ }^{11,12}$. The Dp427 isoform is highly localized in synaptic spines and post-synaptic densities (PSD), where it associates with a dystrophin-associated protein complex (DAPC) which spans the cell membrane and links the actin cytoskeleton with the extracellular matrix ${ }^{13}$. Both spines and PSDs have a critical role for neurotransmission and synaptic plasticity, which underlie cognitive functions, including memory and learning ${ }^{14}$. The cognitive impairment associated with DMD has been linked to the effects of the lack of dystrophin in brain structures associated with memory, language and attention, including the hippocampus and the prefrontal cortex ${ }^{7,15}$. In addition, it should be noted that only one third of DMD patients show cognitive impairment ${ }^{16}$. The cognitive impairment can appear, in the absence of dystrophin, even without the muscular dystrophy symptoms, suggesting that the cognitive symptoms are a direct consequence of the lack of protein, rather than an epiphenomenon paralleling and caused by the motor impairment ${ }^{17}$. Finally, the lack of dystrophin has been associated with epilepsy, as the prevalence of epilepsy in DMD patients is significantly higher in comparison to control populations ${ }^{18-21}$.

81 However, it is not clear yet how the lack of the Dp427 isoform of dystrophin may affect the

82 biophysical properties of membrane conductance and synaptic machinery, responsible for 83 neuronal excitability and synaptic transmission and plasticity, respectively. For example, the 
$m d x$ mouse, the most commonly used mouse model for dystrophin deficiency and DMD, which lacks the expression of the Dp427 kDa dystrophin isoform, has been reported to show impaired spatial memory and enhanced long-term potentiation (LTP) of synaptic transmission in the hippocampus ${ }^{9,22}$, while other research groups have not observed differences in either spatial learning or hippocampal LTP 23 . Intriguingly, motivational differences between the $m d x$ mouse and its wild type control can give conflicting operant conditioning, dependent on the type of behavioural characteristics being assessed ${ }^{24}$. The reasons for all these discrepancies are unclear; however, the absence of a mechanistic pathway leading from the absence of dystrophin to cognitive dysfunction contributes to the lack of consensus on the causal connection between DMD and neuronal function ${ }^{25}$. In addition, such a variety of hippocampal function phenotypes may be associated with the unpredictability of mental retardation onset in people with DMD (only $1 / 3$ of DMD patients show cognitive deficit) ${ }^{16}$.

GABA-receptor density and clustering on post-synaptic membranes, and GABA dependent inhibitory neurotransmission have been shown to be altered in $m d x$ mice. The frequency of GABAergic-dependent spontaneous inhibitory post-synaptic currents (IPSCs) is indeed increased in the amygdala pyramidal neurons of $m d x$ mice, revealing a possible effect of this genotype in promoting the hyperexcitability of inhibitory interneurons in this brain area 9 .

Much less is known about the effect of the loss of dystrophin on basic electrotonic and electrogenic properties of neuronal membranes. In the light of all the above, in this study we investigated the effects of the absence of Dp427 on the excitability of hippocampal CA1 pyramidal neurons as well as the functionality of Schaffer Collaterals - CA1 synapses in DBA/2J-mdx mice. This mouse line has been generated by backcrossing the more commonly used $\mathrm{Bl} / 10-m d x$ mouse line onto a DBA/2J genetic background which results in a more severe pathology of the muscle (ref 37, Coley et al). Although our study was not designed to determine the effect of genetic background on neuronal parameters, we reasoned that the increased muscle weakness in the DBA/2J-mdx mice compared to the $\mathrm{Bl} / 10-m d x$ mice might compound any effects of the lack of dystrophin per se because of additional mobility restrictions in the 
111 mice due to muscle weakness. To the best of our knowledge, no studies have investigated

112 neuronal function in this DMD model.

\section{Materials and methods}

\section{Experimental animals}

116 Male DBA/2J-mdx mice (The Jackson Laboratory, Bar Harbour, ME USA) and DBA2 wild-type

117 mice (Envigo, UK) were group housed in animal facilities at the University of Reading on a

118 12:12 hour light/dark cycle with standard chow and water available at libitum. In all

119 experiments, DBA/2J-mdx mice were compared against age- and sex-matched wild-type

120 mice. All experiments were carried out at the University of Reading under a United Kingdom

121 Home Office licence in compliance with the Animals (Scientific Procedures) Act 1986.

122 Preparation of brain slices.

123 Animals were sacrificed using cervical dislocation in accordance with schedule 1 of the

124 Animals (Scientific Procedures) Act (1986). The brain was rapidly removed and transferred to 125 an ice cold cutting solution consisting of (in mM): 189 Sucrose, 10 D-Glucose, $26 \mathrm{NaHCO}_{3}, 3$ $126 \mathrm{KCl}, 5 \mathrm{MgSO}_{4}\left(7 \mathrm{H}_{2} \mathrm{O}\right), 0.1 \mathrm{CaCl}_{2}, 1.25 \mathrm{NaH}_{2} \mathrm{PO}_{4}$. Three hundred $\mu \mathrm{m}$ coronal sections were 127 cut using a Leica VT1200 microtome and immediately transferred to a holding chamber 128 containing artificial cerebrospinal fluid (aCSF) continuously perfused with carbogen. The 129 composition of the aCSF was as follows (in $\mathrm{mM}$ ): $124 \mathrm{NaCl}, 3 \mathrm{KCl}, 24 \mathrm{NaHCO}_{3}, 2 \mathrm{CaCl}_{2}, 1.25$ $130 \mathrm{NaH}_{2} \mathrm{PO}_{4}, 1 \mathrm{MgSO}_{4}, 10 \mathrm{D}$-glucose. The slices were then allowed to recover for $30 \mathrm{~min}$ at $37^{\circ}$

$131 \mathrm{C}$ and subsequently at room temperature for at least 1 hour prior to transfer into a recording 132 chamber.

133 Whole cell patch clamp recordings

134 Slices were transferred to a recording chamber where they were submerged in carbogen135 equilibrated aCSF and maintained at a temperature between $33-34{ }^{\circ} \mathrm{C}$. The recording 136 chamber was secured on the stage of an Olympus BX51 upright microscope and individual 137 CA1 pyramidal neurons were visualised using infrared differential interference contrast optics. 
138 Borosilicate glass microelectrodes with a resistance ranging from 3-7 $\mathrm{M} \Omega$ were pulled, fire-

139 polished and filled with a K-Gluconate based internal solution consisting of (in $\mathrm{mM}$ ): $120 \mathrm{mM}$

140 K-gluconate, 10 mM Na2-phosphocreatine, 0.3 mM Na2-GTP, 10 mM HEPES, 4 mM KCl, 4

$141 \mathrm{mM}$ Mg-ATP ( $\mathrm{pH} 7.2,280-290$ mOsm). Following entry into whole cell configuration, a junction

142 potential error of $15 \mathrm{mV}$ arose due to the pairing of the pipette solution with the aCSF, which

143 was corrected for arithmetically during analysis. Signals were recorded using a Multiclamp

$144700 \mathrm{~A}$ amplifier, digitised using a Digidata 1550B and stored for future analysis using pClamp

14510 software.

146

147 All recordings were made from a defined pre-stimulus membrane potential set by injecting a

148 continuous flow of bias current through the recording electrode. This facilitated the analysis of

149 passive neuronal properties and action potential generation from pre-stimulus membrane

150 potentials $\left(\mathrm{V}_{\mathrm{m}}\right)$ of both -80 and $-74 \mathrm{mV}$. In order to measure neuronal passive membrane

151 properties a $500 \mathrm{~ms},-100 \mathrm{pA}$ hyperpolarising current was injected across the membrane from

152 each $V_{m}$. The subsequent voltage deflection at the steady state of the hyperpolarisation was

153 used to calculate the input resistance $\left(R_{\text {in }}\right)$ of the membrane using Ohm's law $(V=I R)$. The

154 extrapolation of a single exponential curve at an infinite time, fitted to the membrane charging

155 response between 10 and $95 \%$ of the peak amplitude, was used to calculate the membrane

156 time constant ( $\mathrm{T})$. An approximation of capacitance was measured as the ratio between the $\mathrm{T}$

157 and $\mathrm{R}_{\text {in. }}$.

158 Sag, measured as the difference between the negative peak and the steady state

159 hyperpolarisation, was expressed as a percentage of the peak hyperpolarisation in response

160 to a $500 \mathrm{~ms},-100 \mathrm{pA}$ hyperpolarising current injection.

161 Standard "Zap" protocols were used to measure subthreshold membrane resonance

162 properties. Briefly, the ratio of the Fast-Fourier transform of the voltage response versus the

163 current injection was calculated as a measure of the impedance profile of the pyramidal neurons $\left(Z=V_{\mathrm{fft}} / \mathrm{lfft}_{\mathrm{ft}}\right)$. Subsequently, the impedance versus frequency profile was smoothed 
165 with a 35-point moving average function. The maximal impedance ( $Z$ max) frequency at which

166 this maximal impedance occurred (Peak frequency), and the quality factor of the resonator (Q)

167 were quantified to facilitate comparisons between the genotypes. The quality factor of the

168 resonator "Q" was calculated as the ratio between the impedance at peak frequency and the

169 impedance at $0.5 \mathrm{~Hz}^{26}$.

170 In order to quantify neuronal excitability, a series of incremental $500 \mathrm{~ms}$ depolarising square

171 current injections, ranging from 50 to $350 \mathrm{pA}$, were injected across the membrane. The

172 number of action potentials (AP) generated for each current injection was used as a measure

173 of excitability. The first AP fired in response to a $350 \mathrm{pA}$ depolarising current step was used to

174 compare action potential waveforms between the genotypes. AP threshold was quantified,

175 defined as the voltage at which the rate of rise $(\mathrm{dV} / \mathrm{dt})$ surpassed $20 \mathrm{~V} \mathrm{~s}^{-1}$; the maximal rate of

176 rise (RoR) was also measured for each cell as the highest value of the first derivative of voltage

177 in time, within the AP duration; the AP width was measured at $V_{m}=-15 \mathrm{mV}$; finally, the AP

178 peak was measured as the $V_{m}$ at which, following the ascending phase of the $A P, d V / d t=0$

$179 \mathrm{~V}^{*} \mathrm{~s}^{-1}$

180 For the voltage-clamp experiments, outside-out, somatic, nucleated macropatches were excised as previously described ${ }^{27}$. Pipette capacitance was neutralized and the series

182 resistance was compensated for $(10 \%-80 \%$ correction). VC recordings were made for the

183 quantitative evaluation of outward-going plateau voltage-gated $\mathrm{K}^{+}$currents, by applying $30 \mathrm{~ms}$

184 voltage steps, growing in $10 \mathrm{mV}$ increments and starting from a holding voltage of $-90 \mathrm{mV}$.

185 Each recorded current amplitude was normalized to the membrane capacitance of the 186 macropatch in order to account for differences in current amplitude arising from different sizes 187 of the macropatch. The specific current $(\mathrm{pA} / \mathrm{pF})$ was plotted against the value of the voltage 188 step.

189 Field excitatory postsynaptic potentials

190 Following the initial post-slicing recovery period, the slices were then transferred to a recording 191 chamber in which they were submerged and continually perfused with aCSF, pre-equilibrated 
192 with carbogen and maintained at room temperature. Field excitatory postsynaptic potentials

193 (fEPSPs) were elicited by delivering a short pulse of electrical current $(0.1 \mathrm{~ms})$ through 194 tungsten bipolar stimulating electrodes. These stimulating electrodes were placed such as to 195 stimulate axons of the Schaffer Collateral (SC) pathway. Borosilicate glass recording 196 microelectrodes with a resistance ranging from 3-5 $\mathrm{M} \Omega$ were pulled and filled with aCSF.

197 Recorded signals were collected with a Multiclamp 700A amplifier, digitised with a Digidata

198 1550B and stored for future analysis using pClamp 10 software.

199 Input-output curves were constructed by incrementally increasing the current passing through 200 the stimulating electrode and recording the evoked response $(0-300 \mu \mathrm{A}$ in $50 \mu \mathrm{A})$. For the 201 rest of the experiment, the stimulus intensity was then set to induce $50 \%$ c.a. of the maximal 202 response.

203 Short-term plasticity was tested with a paired-pulse profile, consisting in 2 subsequent stimulating pulses, with inter-pulse intervals of increasing value (in ms): 10, 17, 32, 56, 100, $205170,320,560$, and 1000.

206 After a period of at least 20 minutes recording baseline responses at low frequency $(0.033$ $207 \mathrm{~Hz})$, induction of LTP was attempted in the SC-CA1 pathway using a theta-patterned $(5 \mathrm{~Hz})$ 208 burst stimulation (TBS) protocol. This consisted of 5 bursts of 10 stimuli at $100 \mathrm{~Hz}$ applied with an inter-burst interval of 200 ms. This was repeated 4 times with an inter-repeat interval of 20

210 s. The fEPSPs were then followed for 1 hour before the TBS protocol was delivered to the

211 other pathway and the fEPSPs were followed up for another hour. We have decided to use

212 TBS over other existing LTP-induction protocols, such as high frequency stimulation ${ }^{14}$, as

213 TBS mimics the firing patterns observed in vivo in rodents performing a task leading to long-

214 term memory encoding ${ }^{28-30}$. In addition, TBS results in the most effective NMDA- and voltage215 gated Calcium channel-dependent form of long-lasting LTP ${ }^{31-34}$.

216 Data analysis

217 Data were analysed using Clampfit 11. Statistical assessments of differences between 218 genotypes were made using unpaired two-tailed students t-tests and two-way analysis of 
219 variance (ANOVA) as appropriate. Figures were prepared with Origin Pro 2018. All results

220 were expressed as mean \pm standard error of the mean (SEM). The experimental " $n$ ", refers to

221 the number of slices and to the number of cells we have recorded from in the field potential

222 and patch-clamp experiments, respectively.

Results

225 Most of the studies investigating the functional correlation between the lack of dystrophin and neuronal and brain dysfunction have focused on the alteration of GABAergic neurotransmission. Our study aimed to characterize, in DBA/2J-mdx mice, the effects of the lack of dystrophin on the electrogenic and electrotonic membrane properties of CA1 pyramidal neurons (CA1-PCs). In addition, we investigated whether basal synaptic transmission, shortterm synaptic plasticity and long-term potentiation were affected in DBA/2J-mdx mice. Field potential, extracellular recordings were carried out in $9 \mathrm{~m} / \mathrm{o}$ mice, while single cell, whole-cell patch-clamp recordings were carried out in $7 \mathrm{~m} / \mathrm{o}$ mice. At the chosen age the mice show significant muscle atrophy and weakness (data not shown). In addition, we have observed that DBA/2J-mdx mice show less rearing activity but no changes in other activity related parameters, when tested in activity cages (unpublished observations). Whether this is due to the significant reduction in muscle strength in these mice, or due to reduced motivation to act or explore is not known. Previous studies have shown that the mdx genotype is associated with cognitive impairment ${ }^{35}$. However, to our knowledge, there are no available data on the memory performance in DBA/2J-mdx mice.

240 First, we tested the effect of the DBA/2J-mdx genotype on synaptic transmission (Figure 1A; 241 two-way ANOVA; source of variability: genotype; $F=3.622, P=0.06$ ), short-term plasticity

242 (Figure 1B; two-way ANOVA; source of variability: genotype; $F=0.037, P=0.847$ ) and LTP

243 (Figure 1C; unpaired two-tailed t-test; $\mathrm{P}=0.97$ ). None of these parameters were affected by 244 the DBA/2J-mdx genotype.

245 Synaptic transmission and plasticity are often regarded as the neuronal correlates of cognitive 246 functions, such as memory and learning; hence a change/impairment in these functional 
247 outcomes would be expected to underlie cognitive dysfunction. However, changes in single

248 cell membrane excitability properties are also fundamental functional correlates underlying

249 cognition, even in the absence of synaptic input/output (I/O), (paired-pulse profile) PPP and

250 long-term potentiation (LTP) alterations. For this reason, we measured the electrotonic and

251 electrogenic membrane properties in CA1-PCs from DBA/2J-mdx and age-matched WT

252 controls.

A

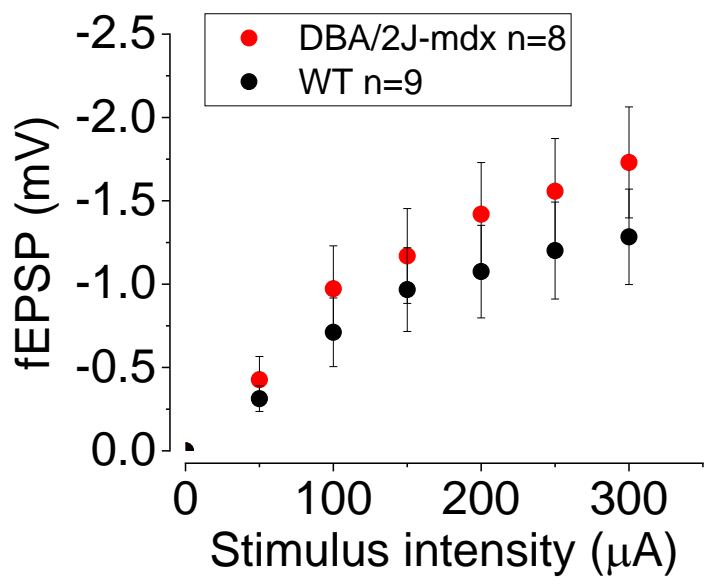

B

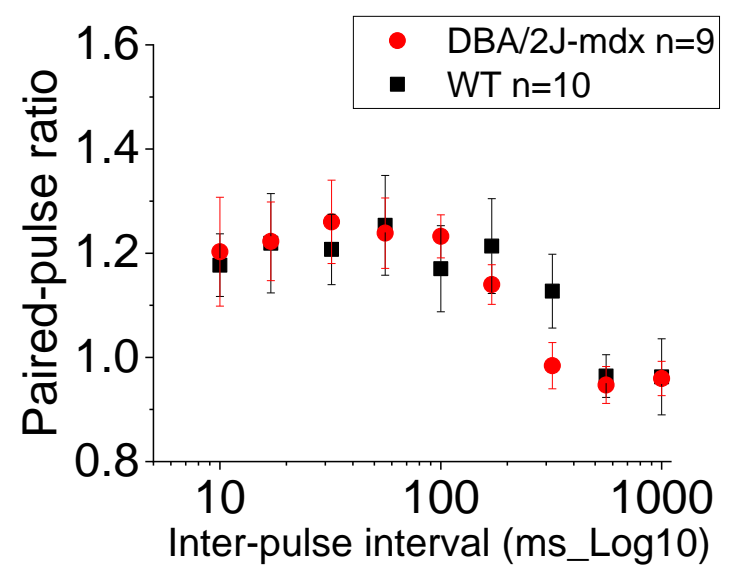

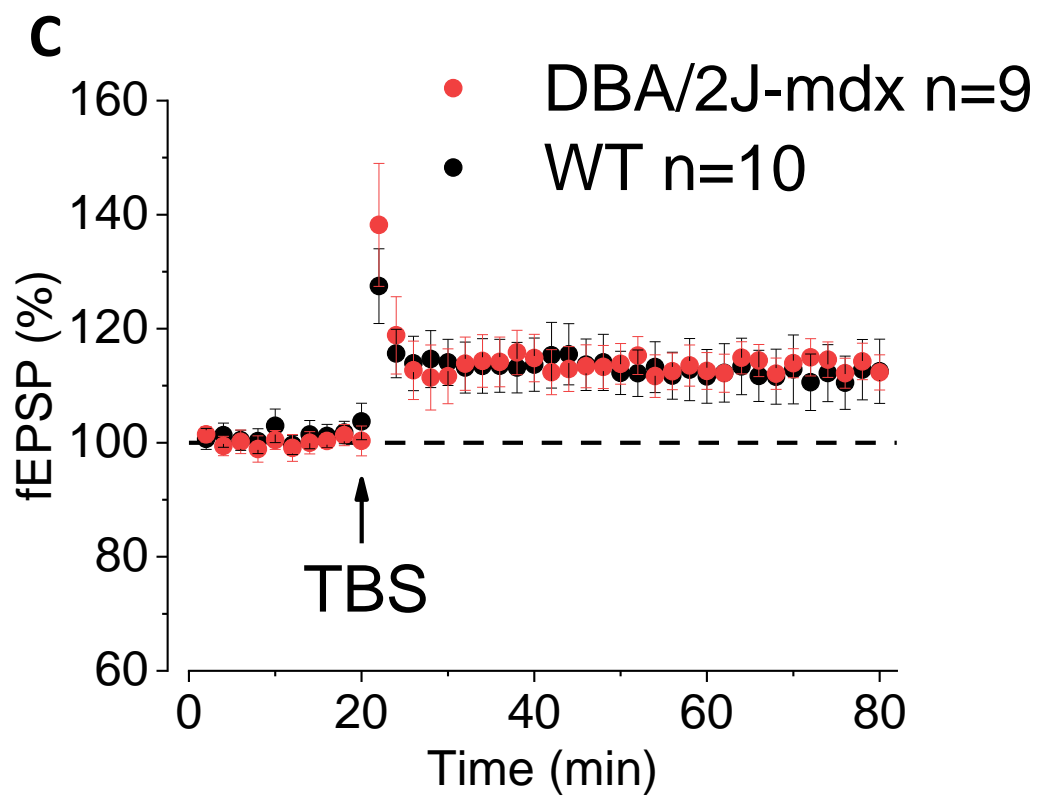


Figure 1. DMD-associated DBA/2J-mdx genotype is not associated with impairments of synaptic transmission and plasticity. A. Basal synaptic transmission was measured as the relationship between fEPSP amplitude and stimulus intensities. Input/output curves were built by plotting the amplitude of SC-CA1 fEPSPs amplitude vs stimulus intensity. Twoway ANOVA does not reveal significant differences between genotypes ( $F=3.622 p=0.06)$ nor interactions between genotype and stimulus intensity $(F=0.104 p=0.991)$. B. Paired pulse ratios of SC-CA1 fEPSPs, tested at increasing inter-pulse intervals, were not different between genotypes (two-way ANOVA; $F=0.037, p=0.847$ ) and no interaction was observed between genotype and ISI (two-way ANOVA; $F=0.499, p=0.855)$. C. LTP induction in SCCA1 synapses was not affected by genotype (Unpaired T-Test on the last 10 minutes of follow-up: $p=0.68$ ). The reported " $n$ " refers to the number of slices.

254 First, no differences were observed in the resting membrane potential (RMP) between

255 genotypes. To avoid biases arising from cell-to-cell variability in RMP, all the other properties

256 were measured from a pre-stimulus potential of $-80 \mathrm{mV}$, obtained with a constant current 
A

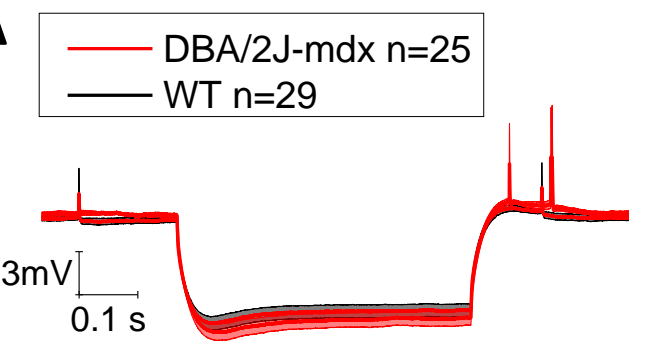

C

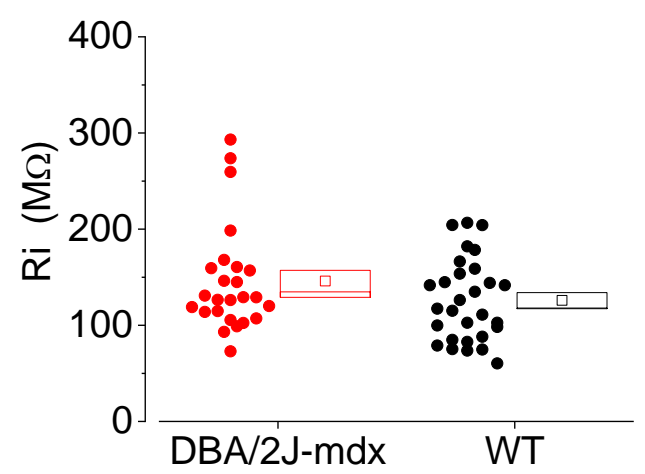

$\mathbf{E}$
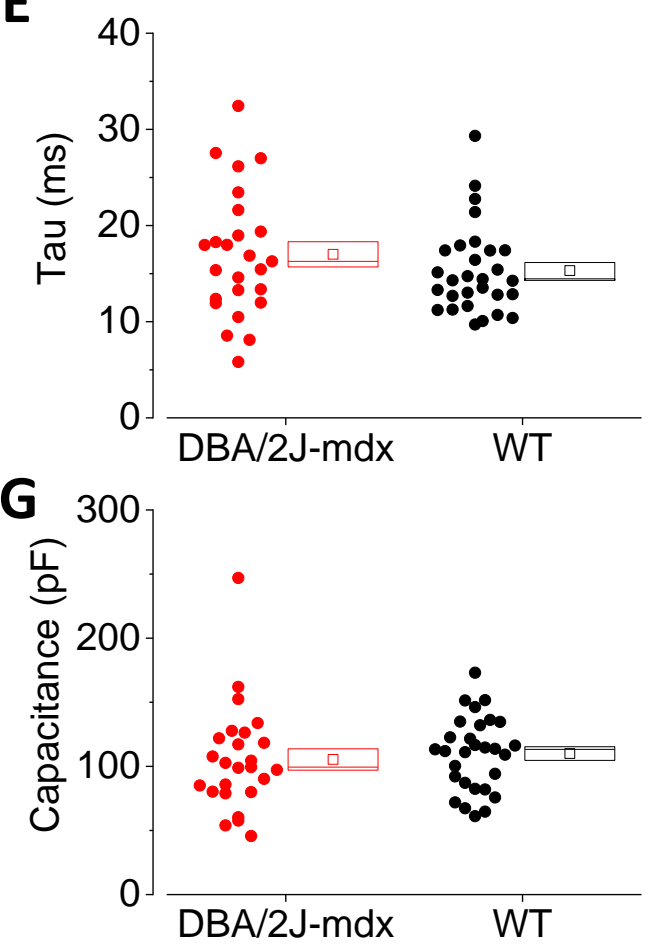
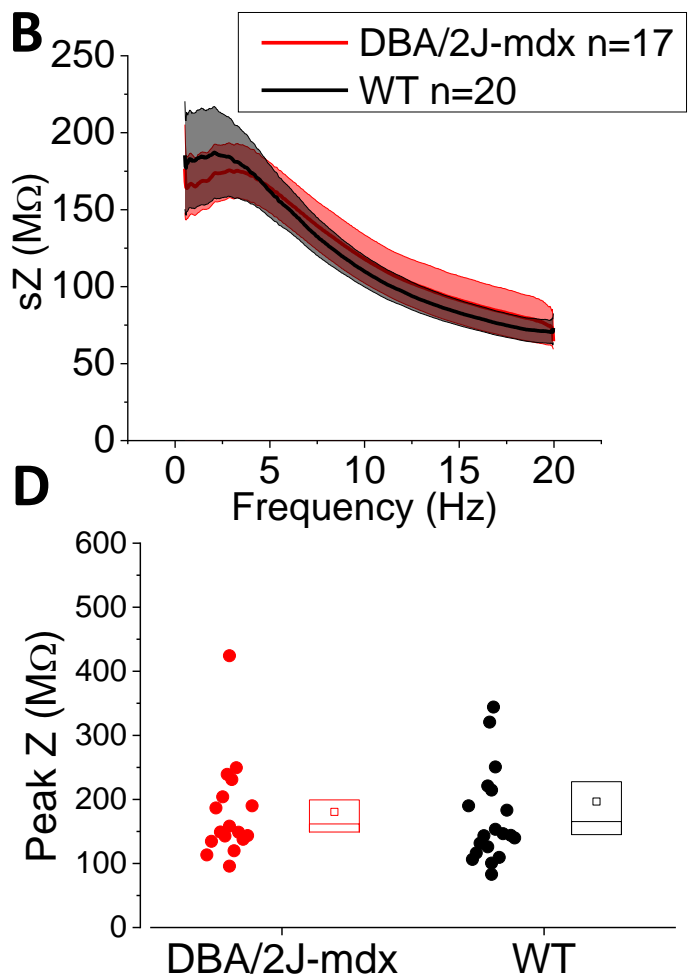

$\mathbf{F}$
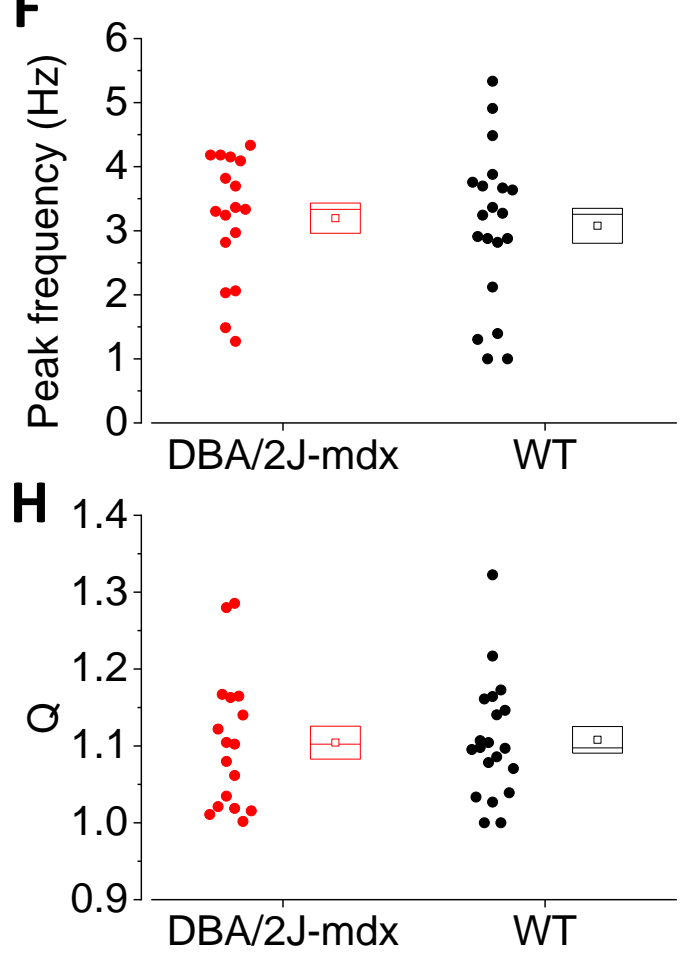

Figure 2. DMD-associated DBA/2J-mdx genotype does not affect the electrotonic, subthreshold membrane properties in hippocampal CA1-PCs. $A$. $V_{m}$ average $\pm S E M$ hyperpolarization, upon the injection of a $-50 \mathrm{pA}, 500 \mathrm{~ms}$ current step injection, for each genotype. This approach was used to measure properties like $R_{\text {in }}(C)$, Tau $(E)$ and capacitance $(G)$, which were not affected by the $m d x$ genotype (unpaired t-test, two tailed). B. Average \pm SEM smoothed impedance (sZ), measured upon the injection of a current oscillating at subthreshold values, with frequencies comprised between $0.5-20 \mathrm{~Hz}$, is plotted here. Resonance properties were measured between genotypes and no effect was observed between $m d x$ mice and WT controls on peak $Z$ (D), peak frequency $(F)$ and quality factor of the resonator $Q(H)$. The reported " $n$ " refers to the number of cells. 
injection. No differences were observed in electrotonic passive properties (Figure 1), neither

259 under the injection of a continuous current (Figure $2 \mathrm{~A}, \mathrm{C}, \mathrm{E}$ and $\mathrm{G}$ ) nor the injection of a 260 subthreshold oscillating current, with a frequency linearly increasing between $0.5 \mathrm{~Hz}$ and 20

$261 \mathrm{~Hz}$ within a $30 \mathrm{~s}$ time window (Figure 2B, D, F, and G). This latter approach was used to 262 investigate the effects of DBA/2J-mdx genotype on the resonance properties of CA1-PCs, represented as Peak Z, Peak Frequency and quality factor of the resonator $-Q$, as previously described ${ }^{36}$. Mean, SEM and P values for subthreshold properties in DBA/2J-mdx and control mice are reported in Table 1.

Table 1. Subthreshold passive and resonance membrane properties of CA1 pyramidal neurons in hippocampal slices from DBA/2J-mdx mice and agematched WT controls.

\begin{tabular}{|c|c|c|c|c|c|}
\hline \multirow[b]{2}{*}{ Property } & \multicolumn{2}{|c|}{ WT $n=29$ cells } & \multicolumn{2}{|c|}{$m d x \mathrm{n}=25$ cells } & \multirow[b]{2}{*}{$\mathbf{P}$} \\
\hline & Average & SEM & Average & SEM & \\
\hline RMP (mV) & $-71.4(n=39)$ & 2.6 & $-73.5(n=33)$ & 3.0 & 0.6 \\
\hline $\operatorname{Rin}(M \Omega)$ & 126.0 & 8.0 & 146.0 & 11.2 & 0.1 \\
\hline $\operatorname{tau}(\mathrm{ms})$ & 15.3 & 0.8 & 17.0 & 1.3 & 0.3 \\
\hline Capacitance (pF) & 110.0 & 5.3 & 105.4 & 8.3 & 0.6 \\
\hline Peak frequency (Hz) & 3.1 & 0.3 & 3.2 & 0.2 & 0.7 \\
\hline $\mathbf{Q}$ & 1.11 & 0.01 & 1.10 & 0.02 & 0.9 \\
\hline Peak Z (M 2$)$ & 196.5 & 31.1 & 180.4 & 18.8 & 0.7 \\
\hline
\end{tabular}

270

272 We tested the firing rate properties of CA1-PCs in DBA/2J-mdx and WT controls upon the 273 injection of square current injections of progressively increasing intensity. The average firing 274 rate was not affected by genotype (Figure 3B; two-way ANOVA, source of Variability: 275 genotype, $F=0.291, P=0.590)$.

276 In addition, the AP waverform properties (Figure 4), namely width (Figure 4C), maximal rate 277 of rise $\left(\mathrm{dV}_{\mathrm{m}} / \mathrm{dT}\right)$ of the AP (RoR) (Figure $\left.4 \mathrm{D}\right)$, peak (Figure 4E) and threshold were not affected 278 by genotype. For averages \pm SEM and $P$ values see Table 2 . 


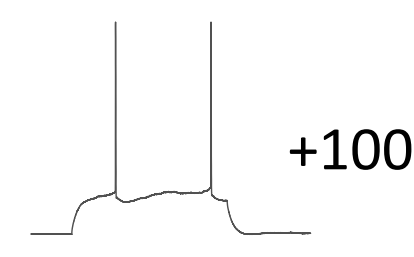

\section{B}
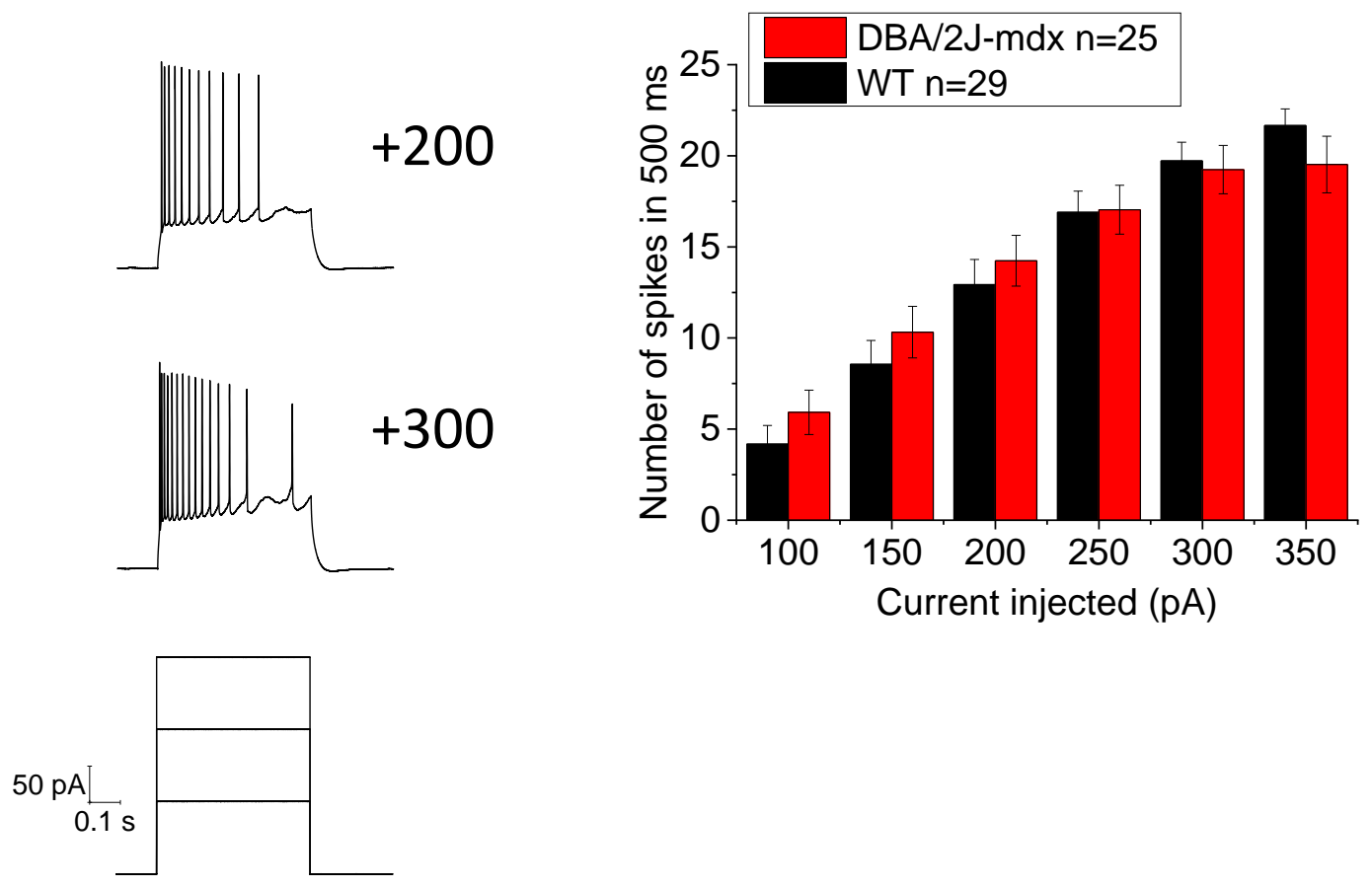

Figure 3. DMD-associated DBA/2J- $m d x$ genotype does not affect the AP firing rates upon depolarization of the $\mathbf{V}_{\mathbf{m}}$, in hippocampal CA1-PCs. A. Example traces of CA1PCs firing APs upon the injection of $100-200-300$ pA, $500 \mathrm{~ms}$, depolarizing current steps. B. The number of APs fired at each current intensity $(50-350 \mathrm{pA}$, with $50 \mathrm{pA}$ increases), within a $500 \mathrm{~ms}$ time window, was plotted as a measure of average firing excitability. No effect of the genotype (two-way ANOVA; $F=0.291, p=0.590$ ) nor interaction between genotype and current intensity (two-way ANOVA; $F=0.745, p=0.590$ ) was observed on CA1-PCs average firing properties. The reported " $n$ " refers to the number of cells.

280 Post-firing properties, such as the medium component of after-hyperpolarisation (mAHP),

281 were tested to investigate the effect of DBA/2J- $m d x$ genotype on the relative refractory

282 period, as an indirect measure of neuronal excitability. mAHPs were evoked by the

283 subsequent application of 5-10-15-20-25, 2 nA-2ms depolarising current steps. The

284 amplitude of the mAHP, comprised of the repolarization of the last evoked AP and the

285 subsequent $500 \mathrm{~ms}$, was measured across number-of-pulses conditions and between genotypes. Figure 5A shows 
A
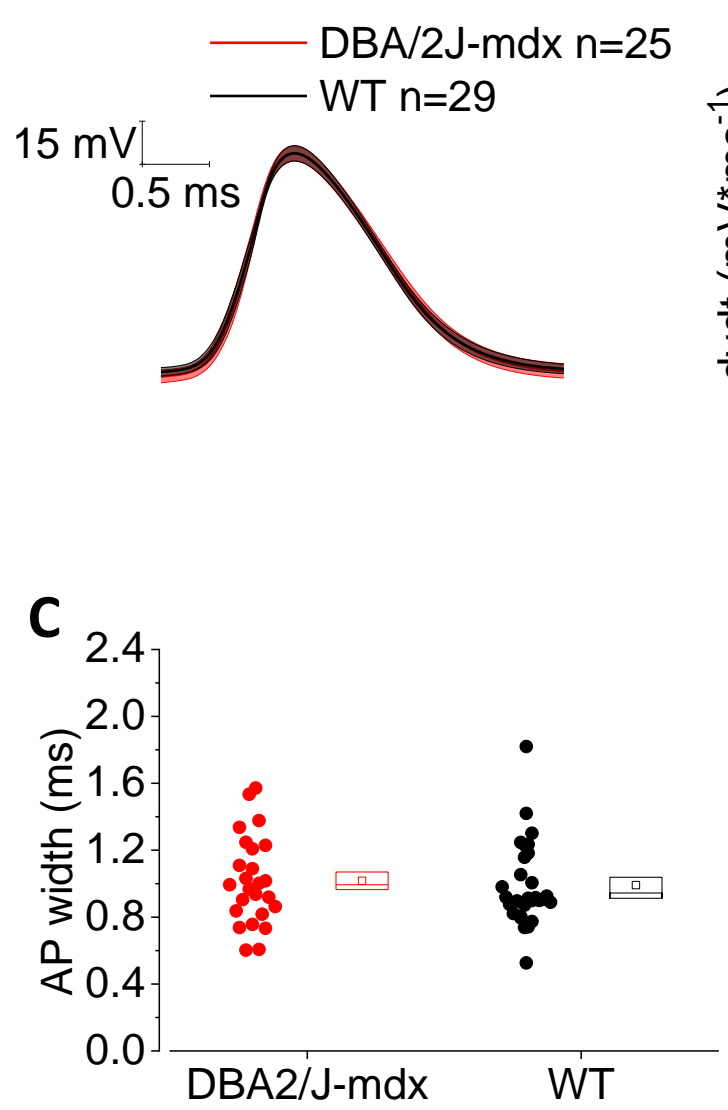

$\mathbf{E}$

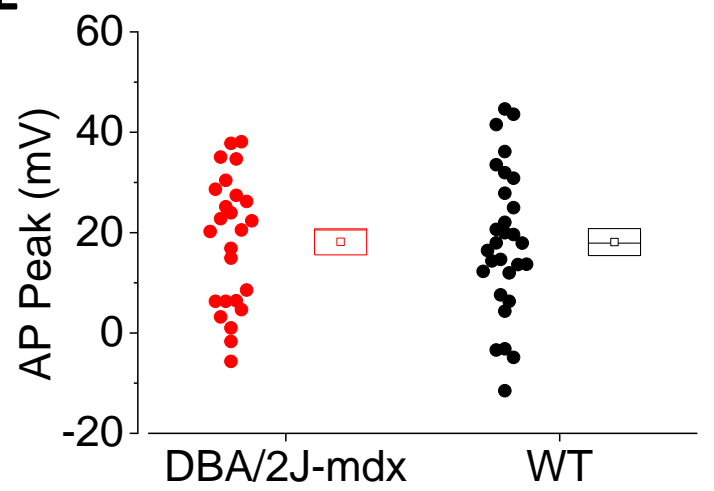

B
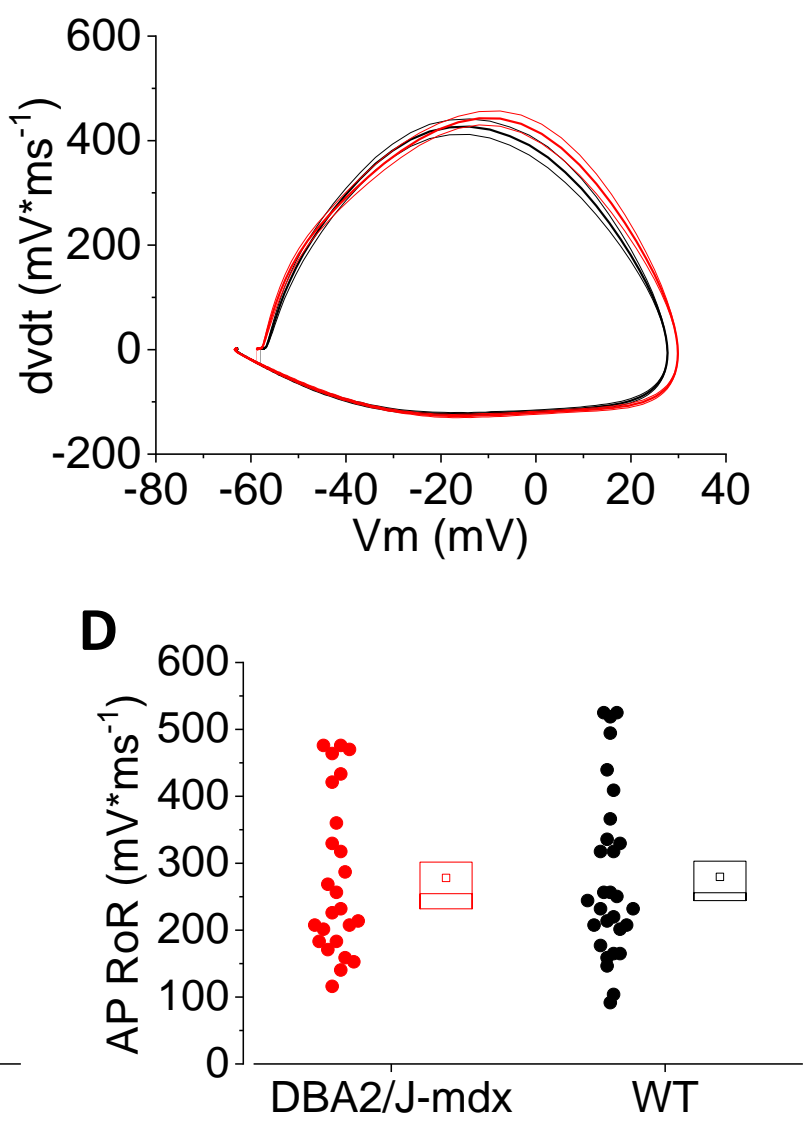

$\mathbf{F}$

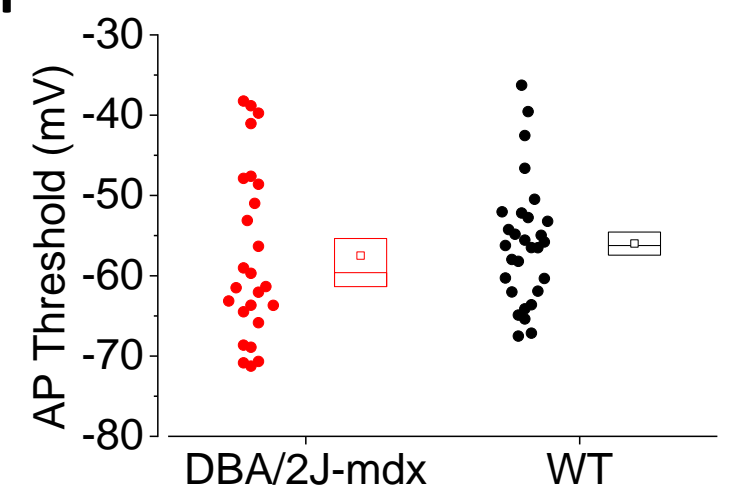

Figure 4. DMD-associated DBA/2J-mdx genotype does not affect the AP waveform properties in hippocampal CA1-PCs. A. Average \pm SEM traces of the first action potential evoked by a 350 pA, 500 ms current step, grouped between genotypes. B. Average \pm SEM phase-plots, grouped between genotypes. CA1-PCs action potential waveform properties, such as width (C), maximal rate of rise (D), peak value (E) and threshold (F) are not different between $m d x$ and WT controls. The reported " $n$ " refers to the number of cells.

the average \pm SEM boundaries of the medium and slow components of the AHP evoked by 20 pulses, while Figure 5B expands the mAHP component, which is visibly bigger in 
291 increasing the mAHP in DBA/2J-mdx mice, compared to WT controls (Figure 5C; two-way

292 ANOVA, source of variability: genotype, $F=9.687, P=0.002$ ).

A

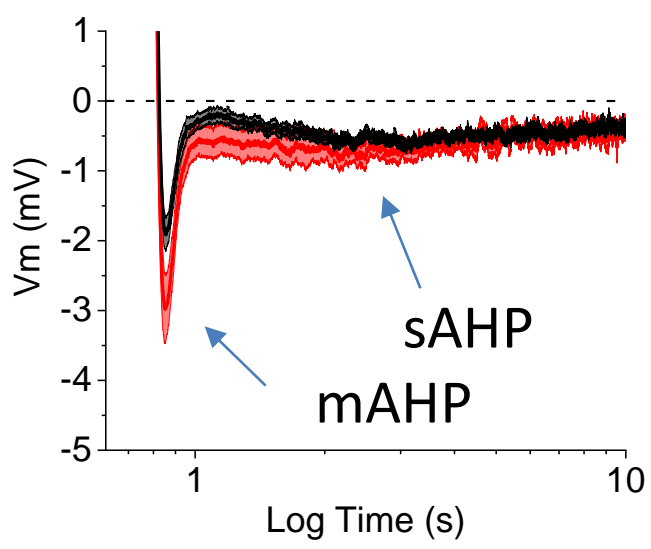

B

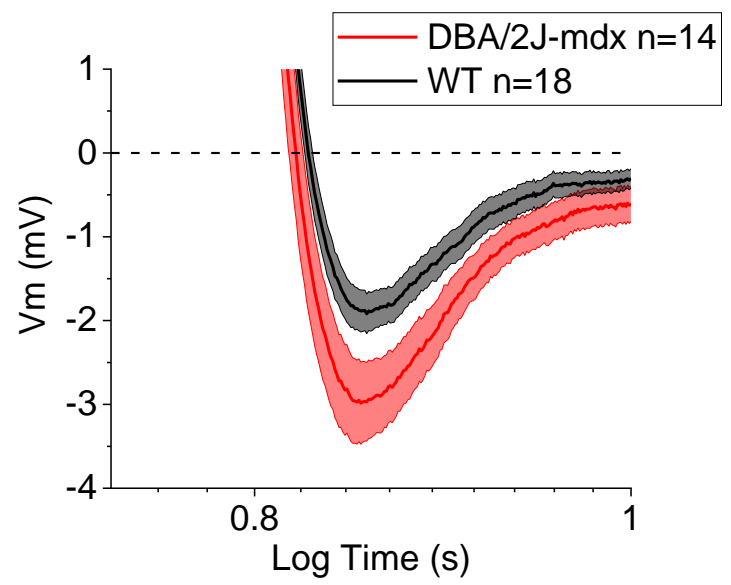

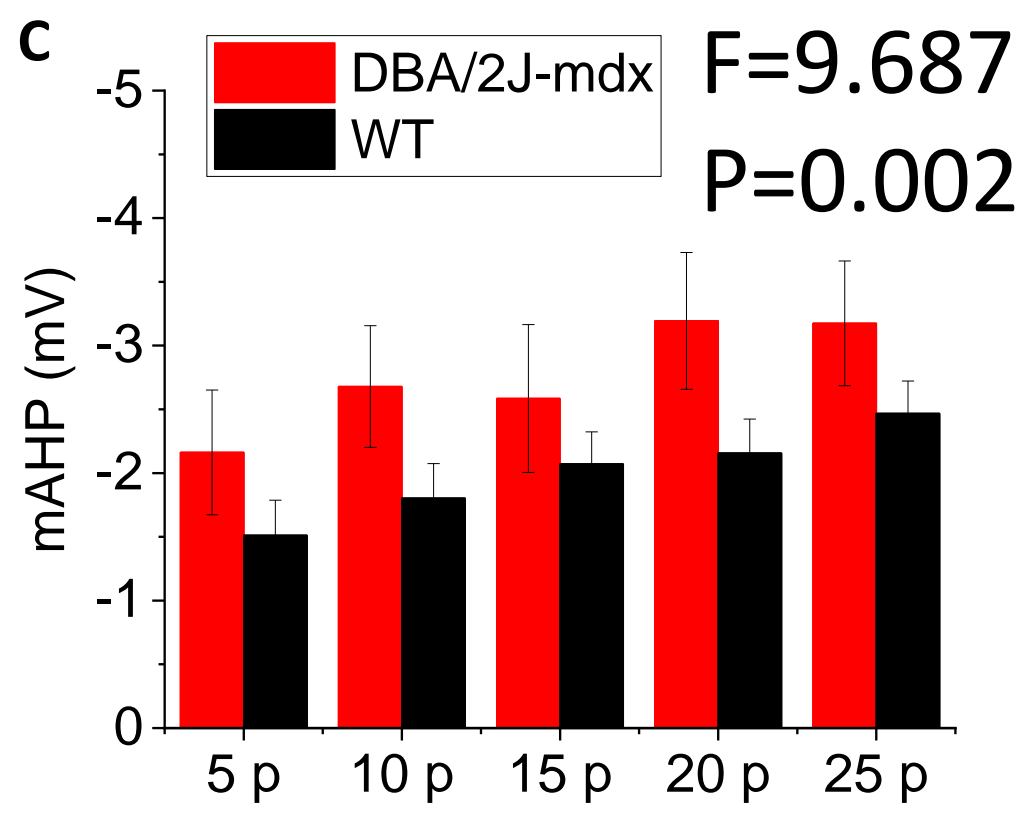

293

Figure 5. DBA/2J-mdx genotype is associated with the increase of the amplitude of post-burst medium but not slow afterhyperpolarizations in hippocampal CA1-PCs. A. Average \pm SEM traces of the mAHP evoked by 20 pulses, $2 \mathrm{nA}-2 \mathrm{~ms}$, delivered at $50 \mathrm{~Hz}$, grouped by genotype. An increased mAHP is observed in CA1-PCs from mdx mice, but no genotype effect was apparent on slow AHP (B). C. The overall mAHP, evoked by 5-10-1520-25 $2 \mathrm{nA} / 2 \mathrm{~ms}$ pulses, delivered at $50 \mathrm{~Hz}$, was bigger in $m d x$ in comparison to WT controls (two-way ANOVA, $F=9.687, p=0.002$ ); however, no interaction was observed between genotype and number of pulses. The reported " $n$ " refers to the number of cells. 
Table 2. Action potential properties of CA1 pyramidal neurons in hippocampal slices from DBA/2J-mdx mice and age-matched WT controls.

297

\begin{tabular}{|c|c|c|c|c|c|}
\hline & \multicolumn{2}{|c|}{ WT $\mathbf{n = 2 9}$ cells } & \multicolumn{2}{c|}{ mdx $\mathbf{n = 2 5}$ cells } & \\
\hline Property & Average & SEM & Average & SEM & P \\
\hline AP_peak (mV) & 18.1 & 2.7 & 18.2 & 2.6 & 1 \\
\hline AP_width (ms) & 0.99 & 0.05 & 1.01 & 0.05 & 0.7 \\
\hline AP_thres (mV) & -56.0 & 1.4 & -57.5 & 2.1 & 0.5 \\
\hline AP_max_dvdt $\left(\mathbf{V s}^{-1}\right)$ & 279.5 & 23.5 & 278.1 & 23.6 & 1 \\
\hline
\end{tabular}

298 The generation of mAHP has been ascribed to the gating properties of non-inactivating, 299 voltage-gated $\mathrm{K}^{+}$currents, such as $\mathrm{I}_{\mathrm{K}}{ }^{36}$. For this reason, we decided to measure the 300 biophysical properties of voltage-gated non-inactivating $\mathrm{I}_{\mathrm{k}}$. Voltage-clamp recordings on

301 nucleated, somatic, outside-out macropatches were performed and cell-to-cell Boltzmann fits were performed to calculate the maximal current density $\left(I_{\max }\right)$ and the half-activation potential $\left(V_{1 / 2}\right)$. However, no significant differences were observed between genotypes for both the $I_{\max }$ (Figure 6; DBA/2J- $m d x I_{\max }=99.7 \mathrm{pA} / \mathrm{pF} \pm 54.4 \mathrm{pA} / \mathrm{pF}$ vs WT $I_{\max }=136.9 \mathrm{pA} / \mathrm{pF} \pm 62.3 \mathrm{pA} / \mathrm{pF}$ $\mathrm{P}=0.7)$ and $\mathrm{V}_{1 / 2}\left(\mathrm{DBA} / 2 \mathrm{~J}-m d x \mathrm{~V}_{1 / 2}=-42.6 \mathrm{mV} \pm 1.9 \mathrm{mV}\right.$ vs $\left.\mathrm{WT} \mathrm{V}_{1 / 2}=-43.3 \mathrm{mV} \pm 1.02 ; \mathrm{P}=0.7\right)$

\section{Discussion}

307 We investigated the effects of the lack of dystrophin on both CA1-PCs intrinsic membrane excitability and SC-CA1 glutamatergic synaptic function in a mouse model of muscular dystrophy, the DBA/2J- $m d x^{37,38}$. The DBA/2J-mdx mouse model, while carrying the same mutation, shows a more severe phenotype, in comparison to $m d x$ mice on a $\mathrm{Bl} / 10$ genetic

311 background ${ }^{37,38}$. In fact, the DBA/2J background contains genetic modifier loci, which are

312 responsible for the increased severity of symptoms observed in the DBA/2J-mdx mice ${ }^{39}$. To

313 our best knowledge, this is the first-time brain function has been tested in this model. Our main 314 observation was that while synaptic function, electrotonic and firing properties were unaffected 315 by the genotype, the mAHP was bigger in DBA/2J- $m d x$ mice than in controls.

316 We have chosen to carry out the recordings on adult mice, aged 7 and 9 -month-old. These 317 ages have been chosen because, while DMD is a disorder mostly affecting people during 
318 development, we were interested to assess the long-term effects of dystrophin deficiency on

319 hippocampal function. In fact, understanding and predicting brain dysfunction associated with

320 DMD, may have a critical role on people's quality of life, should a novel treatment for DMD be

321 developed. While investigating basal synaptic transmission and short-term synaptic plasticity

322 in dystrophin deficient models is novel, LTP has been tested by other groups, showing 323 contrasting results: while some have observed enhanced LTP in dystrophin deficient mice ${ }^{9,22}$,

324 others have not found any effect ${ }^{23}$. Our results fit with the latter. Part of the explanation for 325 the discrepancy 

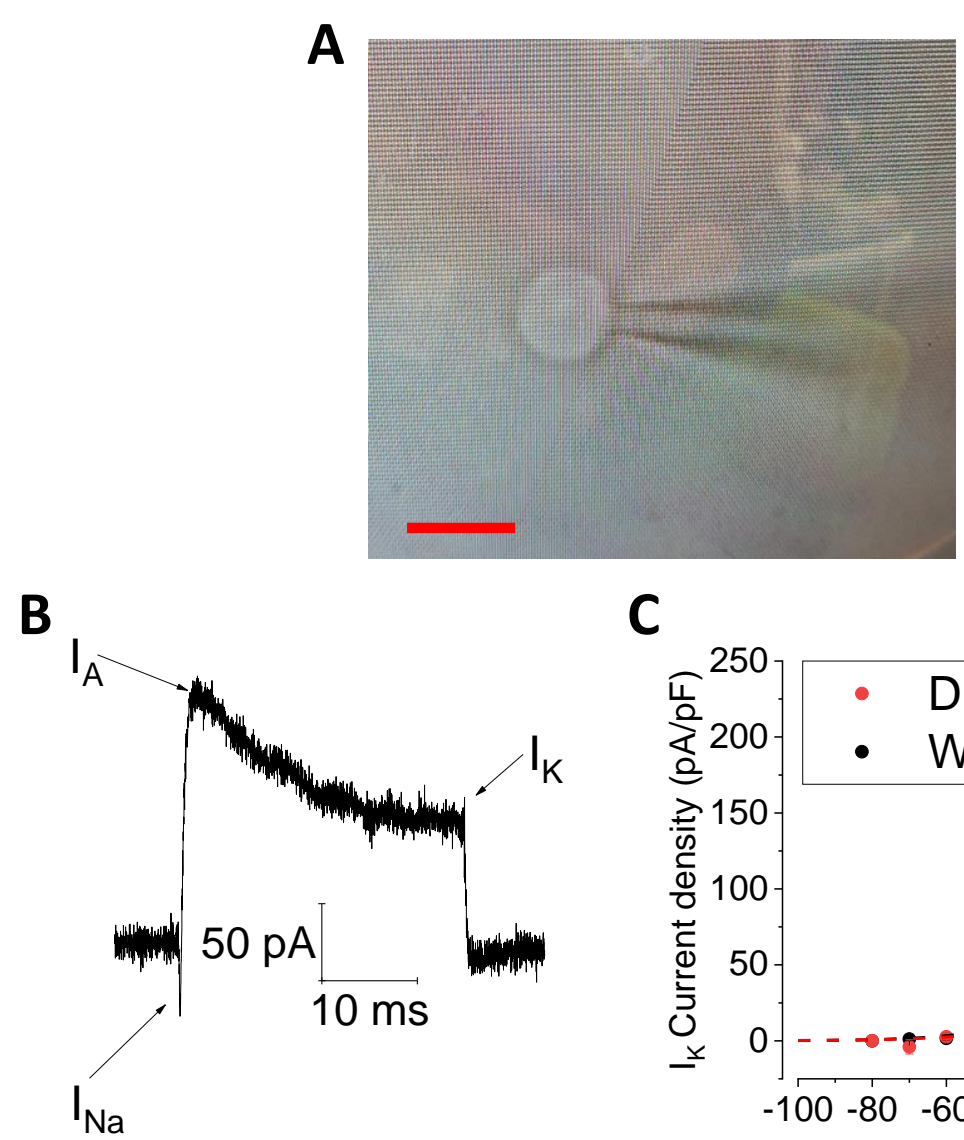

C

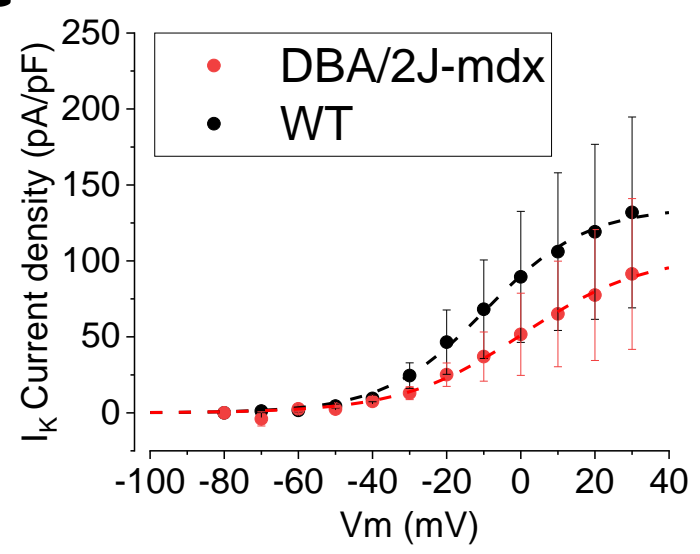

r

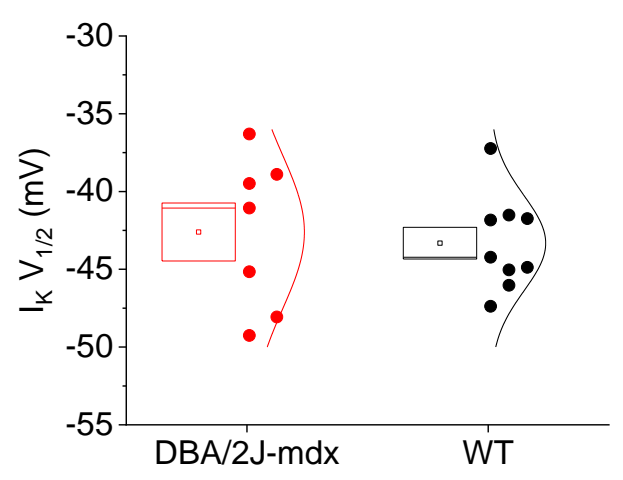

n

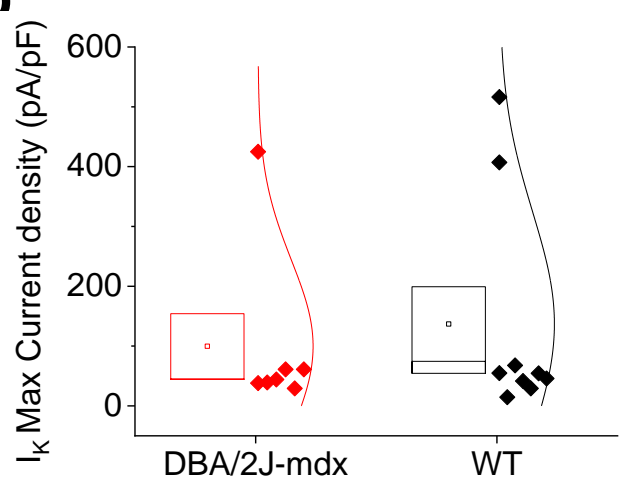

Figure 6. The DBA/2J-mdx genotype does not affect non-inactivating $\mathrm{K}^{+}$currents in CA1-PCs. A. Example of an outside-out nucleated somatic macropatch, pulled from a CA1-PCs at the end of current-clamp recordings. B. Example trace of inward and outward currents evoked by a $+30 \mathrm{mV}$ voltage step: because of the fast inactivating kinetics of $I_{\mathrm{Na}}$ and $I_{A}$, we focused our measures on the inactivating component of voltage-gated outward currents. C. I $I_{K}$ current densities from $m d x$ and WT controls plotted versus the intensity of the voltage step. Cell-to-cell fitting of a Boltzmann function was performed to measure somatic $I_{K}$ maximal current density and $V_{1 / 2}$. Cell-to-cell analysis of the biophysical properties of CA1-PCs' $I_{K}$, revealed no effect of the $m d x$ genotype on somatic $I_{K} V_{1 / 2}(C)$ and maximal current density (D). Scale bar: $5 \mu \mathrm{m}$. 
between the results obtained by different groups may be the different conditions used in each

328 study. First, the LTP-inducing stimulation protocol used in each study is different. In fact, both repeated, strong $(2 \times 1 \mathrm{~s}$ at $100 \mathrm{~Hz}$ every $20 \mathrm{~s})$ and single, weak $(1 \times 1 \mathrm{~s}$ at $30 \mathrm{~Hz})$ high frequency

330 stimulation resulted in a genotype-dependent LTP enhancement in $m d x$ but not in $m d x^{3 \mathrm{cv}}$ mice 331 9,22; however, in vivo LTP experiments on $m d x$ mice, using 3x1s $100 \mathrm{~Hz}$ every $20 \mathrm{~s}$ HFS, did not show any genotype-related change in $m d x$ mice ${ }^{23}$; finally, our experiments were carried out in DBA/2J-mdx mice, using theta-patterned burst $(100 \mathrm{~Hz})$ stimulation. Although experiments were carried out in three different DMD mouse models $\left(m d x, m d x^{3 c v} \& \mathrm{DBA} / 2 \mathrm{~J}-\right.$ $m d x)$ which differ in the severity of the dystrophic pathology, it is currently not possible to conclude whether this influences synaptic plasticity in the brain, because of the use of different protocols by different groups to induce LTP and the fact that dystrophin isoform expression differs between $m d x, m d x^{3 c v}$ and DBA/2J- $m d x$ mice. Given that the muscular pathology of DMD patients is much more severe than that of the $m d x$ mouse, and that muscle-secreted and physical activity-induced factors have an influence on brain neurogenesis and synaptic plasticity ${ }^{40}$ it would be of interest to determine whether severity of muscle pathology affects synaptic plasticity in DMD mouse models. Our data, obtained from the DBA/2J-mdx mouse model, which displays significant muscle atrophy and fibrosis, show no deficit in hippocampal LTP (Figure 1).

Previous work reported that the lack of dystrophin leads to decreased GABAergic neurotransmission ${ }^{41,42}$, due to decreased GABA-A clustering on post-synaptic densities ${ }^{7,43,44}$.

347 Reduced GABAergic neurotransmission has been proposed to be the possible mechanism

348 underlying the increased prevalence of epilepsy in boys with DMD ${ }^{45,46}$ and enhanced 349 hippocampal LTP in DMD mouse models ${ }^{9,47}$. In addition, the Dp71 isoform-dependent 350 dysregulation of the potassium Kir4.1 channel has been proposed as an alternative/complementary mechanism of action responsible for epilepsy in DMD patients.

352 However, it is known that altered synaptic function and epileptiform hypersynchronous 353 network activity also rely on the presence of single cell membrane hyperexcitability ${ }^{27,48-52}$. As 354 we did not observe any change in basal synaptic transmission, short- and long-term synaptic 
plasticity in DBA/2J-mdx mice, we decided to further investigate the effects of lack of

356 dystrophin on the intrinsic physiological properties of the CA1-PC's plasma membrane, as a

357 possible neuronal correlate of DMD-associated brain dysfunction. While electrotonic and firing

358 properties were unchanged between genotypes, the after-hyperpolarization was significantly

359 bigger in DBA/2J-mdx mice. mAHP is mediated by voltage-gated $\mathrm{K}^{+}$channels (VGKC) ${ }^{53}$. To

360 assess the possible role of VGKC for the alteration of mAHP in $m d x$ mice, we performed

361 outside-out nucleated macropatch recordings to assess the biophysical properties of outward

362 voltage-gated currents, as previously reported ${ }^{27,54}$. We did not observe any significant effect

363 of genotype on such current. However, a more detailed investigation of DBA/2J-mdx-

364 dependent alterations of biophysical properties is due, as in our native system we could not

365 tease apart the role of each conductance responsible for voltage-gated $\mathrm{K}^{+}$currents. In fact,

366 dystrophin deficiency has been linked to an array of changes in membrane $\mathrm{K}^{+}$conductances

367 in cardiomyocytes, mediated by different signalling mechanisms. For example, inward rectifier

$368 \mathrm{~K}^{+}\left(\mathrm{K}_{\mathrm{ir}}\right)$ current densities 2.1 were decreased in conditions of dystrophin deficiency ${ }^{55}$ but no

369 changes in the protein channel levels were observed, suggesting a change in trafficking rather

370 than expression. In fact, $\mathrm{K}_{\mathrm{ir}} 2.1$ is functionally and structurally connected to dystrophin via

371 syntrophin ${ }^{56}$ so it has been hypothesized that dystrophin deficiency may result in reduced

372 presence of this conductance on the plasma membrane. However, $K_{v} 2.1$ and 1.5 currents,

373 mediating the slow inactivating component of $\mathrm{I}_{\mathrm{k}}$, are also impaired in cardiomyocytes; they are

374 connected to dystrophin via the F-actin and a-actinin-2 network. For this reason, we

375 hypothesize that, in neurons, the lack of dystrophin may result in increased mAHP via

376 syntrophin mediated altered docking of M-current-generating voltage-gated conductances,

377 such as $K_{v} 7$. In fact, $K_{v} 7 . x$ channels have been reported to mediate the mAHP ${ }^{36}$.

378 While previous work has been carried out on the intrinsic excitability of cerebellar Purkinje

379 cells, showing reduced excitability due to hyperpolarized RMP in dystrophin-deficient mice ${ }^{57}$,

380 such measures have not been performed before in the hippocampus.

381 These observations reinforce the idea that dystrophin may play a key role in brain physiology,

382 regulating both motor and cognitive functions. Several lines of evidence show that dystrophin 
expression is altered in non DMD patients: for example, dystrophin expression is reduced in

384 people with different forms of epilepsy, such as temporal mesial lobe epilepsy ${ }^{58,59}$, sclerotic hippocampus ${ }^{60}$ and in focal cortical dysplasia ${ }^{61}$. However, while the same phenotype has been observed in the piriform cortex of pilocarpine-induced status epilepticus rats rodent models of epilepsy ${ }^{62,63}$, no changes in dystrophin expression have been found in the hippocampus of these animals. On the other hand, dystrophin almost disappeared from the granule cells of the dentate gyrus, following the induction of seizures in mice treated with kainic acid ${ }^{46}$. Such diversity of experimental outcomes somehow reflects the discrepancies observed in the effects of dystrophin deficiency on excitatory synaptic function and it probably reveals the generalized lack of knowledge about the role of this protein on brain physiology. Our results showed increased mAHP in CA1-PCs: this observation may have consequences on network function and cognitive decline observed in people with dystrophin deficiency. In fact, the mAHP corresponds to the refractory period following the AP and has a key role in determining the ability of a single neuron to sustainably fire ${ }^{36}$. An increased mAHP amplitude may result in reduced excitability of the neural network. This, in turn, may account for the cognitive impairment paralleling dystrophin deficiency and be an adaptive mechanism due to increased network excitability. However, the consequences of the increased mAHP in dystrophin deficient mice, both at network and behavioural level, require a more detailed investigation.

It should also be highlighted that while our investigation provides important insights into the alterations of hippocampal neuronal function associated with the lack of Dp427 isoforms, cognitive deficits are more significant in DMD patients which lack expression of shorter dystrophin isoforms derived from downstream promoters on the dystrophin gene, in addition to the full length Dp427 isoform ${ }^{64}$. Whether this is because of a specific role for the shorter dystrophin isoforms or whether this is simply due to the further reduction of the presence of

408 C-terminal dystrophin protein domains in the cell is unknown. However, it is currently not possible to study the role of the shorter dystrophin isoforms in isolation due to the mechanism 
410 by which these different isoforms are generated, but it is possible to isolate the role of the

411 Dp427 isoform.

412 For this reason, we should be cautious in translating these data to all DMD patients.

413 Nonetheless, we believe the results presented here provide insight into the general role of

414 Dp427 in hippocampal function and suggest that the severe muscle dysfunction observed in

415 these mice does not affect most of the CA1 PCs membrane properties.

416 In addition, the analysis of inhibitory interneurons' intrinsic properties in dystrophin deficient

417 mice is required to better clarify the single cell alterations, which may mechanistically explain

418 the effects of dystrophin deficiency on brain function. Finally, clarifying the role of dystrophin

419 in cognition and the pathological effects of its deficiency in brain function may provide evidence

420 of common pathogenic mechanisms between different disorders resulting in cognitive deficit,

421 including DMD, Alzheimer's disease and epilepsy. This may eventually inform the

422 development of a unified model for the onset different brain diseases resulting in impaired 423 cognition.

425 Acknowledgements

426 We thank Erasmus + Traineeship program for funding RB's placement, the University of

427 Reading School of Pharmacy Staff Development and RETF Fund, and Sutura LTD for 428 equipment and consumable expenses.

429 Author contributions

430 RB: extracellular recordings and relative data analysis; WE: experimental design, manuscript

431 revision; FP: experimental design and manuscript revision; LC: experimental design and 432 manuscript revision; HF: experimental design and manuscript revision; KF: scientific direction, 433 experimental design and manuscript revision; FT: scientific direction, intracellular recordings, 434 data analysis, experimental design and manuscript preparation. 
No competing interest

\section{Data availability}

The datasets generated during and/or analysed during the current study are available from

the corresponding author on reasonable request.

References

441

442

1 Hoffman, E. P., Brown, R. H., Jr. \& Kunkel, L. M. Dystrophin: the protein product of

443

444

445

446

447

448

449

450 the Duchenne muscular dystrophy locus. Cell 51, 919-928 (1987).

2 Chamberlain, J. S. et al. Expression of the murine Duchenne muscular dystrophy gene in muscle and brain. Science 239, 1416-1418 (1988).

3 Lidov, H. G., Byers, T. J. \& Kunkel, L. M. The distribution of dystrophin in the murine central nervous system: an immunocytochemical study. Neuroscience 54, 167187 (1993).

4 D'Amario, D. et al. Dystrophin Cardiomyopathies: Clinical Management, Molecular Pathogenesis and Evolution towards Precision Medicine. Journal of clinical medicine

451

452 7, doi:10.3390/jcm7090291 (2018).

453

454

455

456

457

458

459

460

461

462

463

464

465

466

467

468

469

470

471

472

473

474

475

476

477

5 Yiu, E. M. \& Kornberg, A. J. Duchenne muscular dystrophy. Journal of paediatrics and child health 51, 759-764, doi:10.1111/jpc.12868 (2015).

6 Duchenne, G. B. A. Reserches sur la paralysie musculaire pseudeohyper-trofique, ou paralysie myo-sclerosique. Arch. Gen. Med. 11, 5-25, 179-209,305-121, 421-143, 552-188. (1868).

7 Anderson, J. L., Head, S. I., Rae, C. \& Morley, J. W. Brain function in Duchenne muscular dystrophy. Brain : a journal of neurology 125, 4-13 (2002).

8 Cyrulnik, S. E. \& Hinton, V. J. Duchenne muscular dystrophy: a cerebellar disorder? Neurosci Biobehav Rev 32, 486-496, doi:10.1016/j.neubiorev.2007.09.001 (2008).

9 Vaillend, C., Billard, J. M. \& Laroche, S. Impaired long-term spatial and recognition memory and enhanced CA1 hippocampal LTP in the dystrophin-deficient Dmd(mdx) mouse. Neurobiol Dis 17, 10-20, doi:10.1016/j.nbd.2004.05.004 (2004).

10 Doorenweerd, N. et al. Timing and localization of human dystrophin isoform expression provide insights into the cognitive phenotype of Duchenne muscular dystrophy. Scientific reports 7, 12575-12575, doi:10.1038/s41598-017-12981-5 (2017).

11 Lidov, H. G., Selig, S. \& Kunkel, L. M. Dp140: a novel 140 kDa CNS transcript from the dystrophin locus. Hum Mol Genet 4, 329-335 (1995).

12 de Brouwer, A. P. et al. A 3-base pair deletion, c.9711_9713del, in DMD results in intellectual disability without muscular dystrophy. European journal of human genetics : EJHG 22, 480-485, doi:10.1038/ejhg.2013.169 (2014).

13 Kim, T. W., Wu, K., Xu, J. L. \& Black, I. B. Detection of dystrophin in the postsynaptic density of rat brain and deficiency in a mouse model of Duchenne muscular dystrophy. Proc Natl Acad Sci U S A 89, 11642-11644 (1992).

14 Bliss, T. V. \& Collingridge, G. L. A synaptic model of memory: long-term potentiation in the hippocampus. Nature 361, 31-39, doi:10.1038/361031a0 (1993). 
15 Waite, A., Tinsley, C. L., Locke, M. \& Blake, D. J. The neurobiology of the dystrophin-associated glycoprotein complex. Annals of medicine 41, 344-359, doi:10.1080/07853890802668522 (2009).

16 Anand, A. et al. Dystrophin induced cognitive impairment: mechanisms, models and therapeutic strategies. Annals of neurosciences 22, 108-118, doi:10.5214/ans.0972.7531.221210 (2015).

17 de Brouwer, A. P. M. et al. A 3-base pair deletion, c.9711_9713del, in DMD results in intellectual disability without muscular dystrophy. European journal of human genetics : EJHG 22, 480-485, doi:10.1038/ejhg.2013.169 (2014).

18 Goodwin, F., Muntoni, F. \& Dubowitz, V. Epilepsy in Duchenne and Becker muscular dystrophies. Eur J Paediatr Neurol 1, 115-119 (1997).

19 Etemadifar, M. \& Molaei, S. Epilepsy in Boys with Duchenne Muscular Dystrophy Journal of Research in Medical Sciences 3, 116-119 (2004).

20 Pane, M. et al. Duchenne muscular dystrophy and epilepsy. Neuromuscul Disord 23, 313-315, doi:10.1016/j.nmd.2013.01.011 (2013).

21 Hendriksen, R. G. et al. A possible role of dystrophin in neuronal excitability: a review of the current literature. Neurosci Biobehav Rev 51, 255-262, doi:10.1016/j.neubiorev.2015.01.023 (2015).

22 Vaillend, C. et al. Spatial discrimination learning and CA1 hippocampal synaptic plasticity in $\mathrm{mdx}$ and $\mathrm{mdx} 3 \mathrm{cv}$ mice lacking dystrophin gene products. Neuroscience 86, 53-66 (1998).

23 Sesay, A. K., Errington, M. L., Levita, L. \& Bliss, T. V. Spatial learning and hippocampal long-term potentiation are not impaired in mdx mice. Neuroscience letters 211, 207-210 (1996).

24 Lewon, M. et al. Evaluation of the behavioral characteristics of the mdx mouse model of duchenne muscular dystrophy through operant conditioning procedures. Behavioural processes 142, 8-20, doi:10.1016/j.beproc.2017.05.012 (2017).

25 Wikiera, B., Jakubiak, A., Zimowski, J., Noczynska, A. \& Smigiel, R. Complex glycerol kinase deficiency - X-linked contiguous gene syndrome involving congenital adrenal hypoplasia, glycerol kinase deficiency, muscular Duchenne dystrophy and intellectual disability (IL1RAPL gene deletion). Pediatric endocrinology, diabetes, and metabolism 18, 153-157 (2012).

26 Vazifehkhah Ghaffari, B., Kouhnavard, M., Aihara, T. \& Kitajima, T. Mathematical modeling of subthreshold resonant properties in pyloric dilator neurons. BioMed research international 2015, 135787, doi:10.1155/2015/135787 (2015).

27 Brown, J. T., Chin, J., Leiser, S. C., Pangalos, M. N. \& Randall, A. D. Altered intrinsic neuronal excitability and reduced $\mathrm{Na}$ c currents in a mouse model of Alzheimer's disease. Neurobiology of aging 32, 2109 e2101-2114, doi:10.1016/j.neurobiolaging.2011.05.025 (2011).

28 Ranck, J. B., Jr. Studies on single neurons in dorsal hippocampal formation and septum in unrestrained rats. I. Behavioral correlates and firing repertoires. Experimental neurology 41, 461-531 (1973).

29 Otto, T., Eichenbaum, H., Wiener, S. I. \& Wible, C. G. Learning-related patterns of CA1 spike trains parallel stimulation parameters optimal for inducing hippocampal long-term potentiation. Hippocampus 1, 181-192, doi:10.1002/hipo.450010206 (1991).

30 Hill, A. J. First occurrence of hippocampal spatial firing in a new environment. Experimental neurology 62, 282-297, doi:https://doi.org/10.1016/00144886(78)90058-4 (1978). 
31 Grover, L. M. \& Teyler, T. J. Two components of long-term potentiation induced by different patterns of afferent activation. Nature 347, 477-479, doi:10.1038/347477a0 (1990).

32 Grover, L. M. \& Teyler, T. J. Activation of NMDA receptors in hippocampal area CA1 by low and high frequency orthodromic stimulation and their contribution to induction of long-term potentiation. Synapse (New York, N.Y.) 16, 66-75, doi:10.1002/syn.890160108 (1994).

33 O'Connor, J. J., Rowan, M. J. \& Anwyl, R. Long-lasting enhancement of NMDA receptor-mediated synaptic transmission by metabotropic glutamate receptor activation. Nature 367, 557-559, doi:10.1038/367557a0 (1994).

34 O'Leary, D. M. \& O'Connor, J. J. Potentiation of synaptic transmission by (S)-3,5dihydroxy phenylglycine in the rat dentate gyrus in vitro: a role for voltage dependent calcium channels and protein kinase C. Progress in neuro-psychopharmacology \& biological psychiatry 23, 133-147 (1999).

35 Comim, C. M. et al. Neurocognitive Impairment in mdx Mice. Molecular Neurobiology 56, 7608-7616, doi:10.1007/s12035-019-1573-7 (2019).

$36 \mathrm{Gu}$, N., Vervaeke, K., Hu, H. \& Storm, J. F. Kv7/KCNQ/M and HCN/h, but not $\mathrm{KCa} 2 / \mathrm{SK}$ channels, contribute to the somatic medium after-hyperpolarization and excitability control in CA1 hippocampal pyramidal cells. J Physiol 566, 689-715, doi:10.1113/jphysiol.2005.086835 (2005).

37 Coley, W. D. et al. Effect of genetic background on the dystrophic phenotype in mdx mice. Hum Mol Genet 25, 130-145, doi:10.1093/hmg/ddv460 (2016).

38 Hakim, C. H. et al. A Five-Repeat Micro-Dystrophin Gene Ameliorated Dystrophic Phenotype in the Severe DBA/2J-mdx Model of Duchenne Muscular Dystrophy. Mol Ther Methods Clin Dev 6, 216-230, doi:10.1016/j.omtm.2017.06.006 (2017).

39 Coley, W. D. et al. Effect of genetic background on the dystrophic phenotype in mdx mice. Hum Mol Genet 25, 130-145, doi:10.1093/hmg/ddv460 (2016).

40 Lourenco, M. V. et al. Exercise-linked FNDC5/irisin rescues synaptic plasticity and memory defects in Alzheimer's models. Nat Med 25, 165-175, doi:10.1038/s41591018-0275-4 (2019).

41 Kueh, S. L., Head, S. I. \& Morley, J. W. GABA(A) receptor expression and inhibitory post-synaptic currents in cerebellar Purkinje cells in dystrophin-deficient mdx mice. Clin Exp Pharmacol Physiol 35, 207-210, doi:10.1111/j.1440-1681.2007.04816.x (2008).

42 Nusser, Z., Cull-Candy, S. \& Farrant, M. Differences in synaptic GABA(A) receptor number underlie variation in GABA mini amplitude. Neuron 19, 697-709 (1997).

43 Vaillend, C. et al. Rescue of a dystrophin-like protein by exon skipping in vivo restores GABAA-receptor clustering in the hippocampus of the mdx mouse. Mol Ther 18, 1683-1688, doi:10.1038/mt.2010.134 (2010).

44 Waite, A., Brown, S. C. \& Blake, D. J. The dystrophin-glycoprotein complex in brain development and disease. Trends Neurosci 35, 487-496, doi:10.1016/j.tins.2012.04.004 (2012).

45 Nakao, K., Kito, S., Muro, T., Tomonaga, M. \& Mozai, T. Nervous system involvement in progressive muscular dystrophy. Proc Aust Assoc Neurol 5, 557-564 (1968).

46 Knuesel, I., Zuellig, R. A., Schaub, M. C. \& Fritschy, J. M. Alterations in dystrophin and utrophin expression parallel the reorganization of GABAergic synapses in a mouse model of temporal lobe epilepsy. Eur J Neurosci 13, 1113-1124 (2001).

47 Vaillend, C., Ungerer, A. \& Billard, J. M. Facilitated NMDA receptor-mediated synaptic plasticity in the hippocampal CA1 area of dystrophin-deficient mice. 
602

603

604

605

606

607

608

609

610

611

612

613

614

615

616

617

618

619

620

621

622

623

624

Synapse 33, 59-70, doi:10.1002/(sici)1098-2396(199907)33:1<59::aid-syn6>3.0.co;2$\mathrm{k}(1999)$.

48 Palop, J. J. et al. Aberrant excitatory neuronal activity and compensatory remodeling of inhibitory hippocampal circuits in mouse models of Alzheimer's disease. Neuron 55, 697-711, doi:10.1016/j.neuron.2007.07.025 (2007).

49 Tamagnini, F. et al. Altered intrinsic excitability of hippocampal CA1 pyramidal neurons in aged PDAPP mice. Frontiers in cellular neuroscience 9, 372, doi:10.3389/fncel.2015.00372 (2015).

50 Tamagnini, F., Scullion, S., Brown, J. T. \& Randall, A. D. Intrinsic excitability changes induced by acute treatment of hippocampal CA1 pyramidal neurons with exogenous amyloid beta peptide. Hippocampus 25, 786-797, doi:10.1002/hipo.22403 (2015).

51 Kerrigan, T. L. \& Randall, A. D. A new player in the "synaptopathy" of Alzheimer's disease - arc/arg 3.1. Front Neurol 4, 9, doi:10.3389/fneur.2013.00009 (2013).

52 Randall, A. D., Witton, J., Booth, C., Hynes-Allen, A. \& Brown, J. T. The functional neurophysiology of the amyloid precursor protein (APP) processing pathway. Neuropharmacology 59, 243-267, doi:10.1016/j.neuropharm.2010.02.011 (2010).

$53 \mathrm{Gu}, \mathrm{N}$., Vervaeke, K., Hu, H. \& Storm, J. F. Kv7/KCNQ/M and HCN/h, but not $\mathrm{KCa} 2 / \mathrm{SK}$ channels, contribute to the somatic medium after-hyperpolarization and excitability control in CA1 hippocampal pyramidal cells. The Journal of physiology 566, 689-715, doi:10.1113/jphysiol.2005.086835 (2005).

54 Tamagnini, F. et al. Hippocampal neurophysiology is modified by a diseaseassociated C-terminal fragment of tau protein. Neurobiology of aging 60, 44-56, doi:10.1016/j.neurobiolaging.2017.07.005 (2017).

55 Rubi, L., Koenig, X., Kubista, H., Todt, H. \& Hilber, K. Decreased inward rectifier potassium current IK1 in dystrophin-deficient ventricular cardiomyocytes. Channels (Austin, Tex.) 11, 101-108, doi:10.1080/19336950.2016.1228498 (2017).

56 Willis, B. C., Ponce-Balbuena, D. \& Jalife, J. Protein assemblies of sodium and inward rectifier potassium channels control cardiac excitability and arrhythmogenesis. American journal of physiology. Heart and circulatory physiology 308, H1463-1473, doi:10.1152/ajpheart.00176.2015 (2015).

57 Snow, W. M., Anderson, J. E. \& Fry, M. Regional and genotypic differences in intrinsic electrophysiological properties of cerebellar Purkinje neurons from wild-type and dystrophin-deficient mdx mice. Neurobiol Learn Mem 107, 19-31, doi:10.1016/j.nlm.2013.10.017 (2014).

58 Lee, T. S. et al. Aquaporin-4 is increased in the sclerotic hippocampus in human temporal lobe epilepsy. Acta Neuropathol 108, 493-502, doi:10.1007/s00401-0040910-7 (2004).

59 Das, A. et al. Hippocampal tissue of patients with refractory temporal lobe epilepsy is associated with astrocyte activation, inflammation, and altered expression of channels and receptors. Neuroscience 220, 237-246, doi:10.1016/j.neuroscience.2012.06.002 (2012).

60 Eid, T. et al. Loss of perivascular aquaporin 4 may underlie deficient water and $\mathrm{K}+$ homeostasis in the human epileptogenic hippocampus. Proc Natl Acad Sci U S A 102, 1193-1198, doi:10.1073/pnas.0409308102 (2005).

61 Medici, V., Frassoni, C., Tassi, L., Spreafico, R. \& Garbelli, R. Aquaporin 4 expression in control and epileptic human cerebral cortex. Brain Res 1367, 330-339, doi:10.1016/j.brainres.2010.10.005 (2011). 
62562 Kim, J. E. et al. Astroglial loss and edema formation in the rat piriform cortex and hippocampus following pilocarpine-induced status epilepticus. J Comp Neurol 518, 4612-4628, doi:10.1002/cne.22482 (2010).

63 Sheen, S. H. et al. Decrease in dystrophin expression prior to disruption of brainblood barrier within the rat piriform cortex following status epilepticus. Brain Res 1369, 173-183, doi:10.1016/j.brainres.2010.10.080 (2011).

64 Taylor, P. J. et al. Dystrophin gene mutation location and the risk of cognitive impairment in Duchenne muscular dystrophy. PLoS One 5, e8803, doi:10.1371/journal.pone.0008803 (2010). 
